\title{
REDUÇÃO DO CONSUMO DE VAPOR NA OPERAÇÃO DE TORRES DE RECUPERAÇÃO DE AMÔNIA UTILIZAANDO CONTROLE AVANÇADO BASEADO EM LÓGICA FUZZY*
}

\author{
Rodrigo Alberto Moreira Gomes ${ }^{1}$ \\ Luciano Sant Ana Pereira ${ }^{2}$ \\ Pedro Baião Júnior ${ }^{2}$ \\ Flávio Seara Ramos ${ }^{2}$ \\ Ronaldo Pereira Caixeta ${ }^{2}$ \\ Admir José e Silva ${ }^{2}$ \\ Igor Santiago ${ }^{3}$ \\ Leonardo Freitas ${ }^{3}$ \\ Sayonara Soares ${ }^{3}$
}

\section{Resumo}

Para a purificação do níquel laterítico explorado na mina localizada em Niquelândia/GO, pela Votorantim Metais Níquel (VMN), utiliza-se solução amoniacal durante a etapa de lixiviação do minério, seguindo a rota hidrometalúrgica de produção. A amônia é recuperada em torres de destilação por arraste com vapor vivo, para ser reutilizada em um novo ciclo de produção. Este trabalho apresenta a aplicação de uma tecnologia baseada em lógica Fuzzy no conjunto de 6 torres de recuperação de amônia, com o objetivo de controlar as alimentações de solução a ser purificada e de vapor. A plataforma de controle avançado utilizada, Leaf, foi desenvolvida pela I.Systems Automação Industrial, empresa localizada em Campinas/SP, e não necessita da criação de modelos matemáticos fenomenológicos para geração e sintonia dos controladores. Com a automatização da operação das torres, o consumo de vapor da unidade foi reduzido em $3,5 \%$ e a intervenção manual foi reduzida.

Palavras-chave: Torre de amônia; Controle avançado; Lógica Fuzzy; Níquel.

\section{REDUCED CONSUMPTION OF STEAM IN THE TOWERS OF OPERATION AMMONIA RECOVERY USING ADVANCED CONTROL BASED ON FUZZY LOGIC}

\section{Abstract saved and manual intervention was reduced. \\ Votorantim Metais, Belo Horizonte, Minas Gerais, Brasil. \\ Votorantim Metais, Niquelândia, Goiás, Brasil. \\ I.Systems, Campinas, São Paulo, Brasil.}

Votorantim Metais Níquel (VMN) purifies lateritic nickel exploited in Niquelândia's mine, a city located in the state of Goiás, Brazil, using ammonia solution during the ore leaching step, following the hydrometallurgical production route. The ammonia is recovered in steam stripping towers, to be reused in a new production cycle. This paper presents the application of a technology based on Fuzzy logic on six towers, in order to control the solution to be purified and the steam usage. The advanced platform control used, Leaf, was developed by I.Systems Industrial Automation, a company located in Campinas/SP, and does not require the creation of phenomenological mathematical models for generation and tuning of process controllers. By automating the towers' operation, 3.5\% of the steam consumption was

Keywords: Ammonia stripping towers; Advanced control; Fuzzy logic; Nickel. 


\section{INTRODUÇÃO}

A Votorantim Metais Níquel (VMN), localizada em Niquelândia/GO, produz concentrado de níquel/cobalto na forma de carbonato, para ser posteriormente processado na unidade industrial de São Miguel Paulista, em São Paulo/SP.

O minério de níquel passa por uma série de operações unitárias de beneficiamento físico e de redução, até chegar à etapa de lixiviação por amônia. Nesta última etapa, níquel, cobre e cobalto são solubilizados e seguem para a destilação, etapa que gera uma solução amoniacal e lama residual. A solução alimenta uma torre de destilação por arraste a vapor, equipamento no qual ocorre transferência de massa da fase líquida para a fase gasosa. $O$ vapor, alimentado na base da torre em contracorrente com a solução, volatiliza a amônia e arrasta-a para o topo, para ao final ser absorvida em outros processos, gerando nova solução primária, fechando o ciclo de utilização deste insumo.

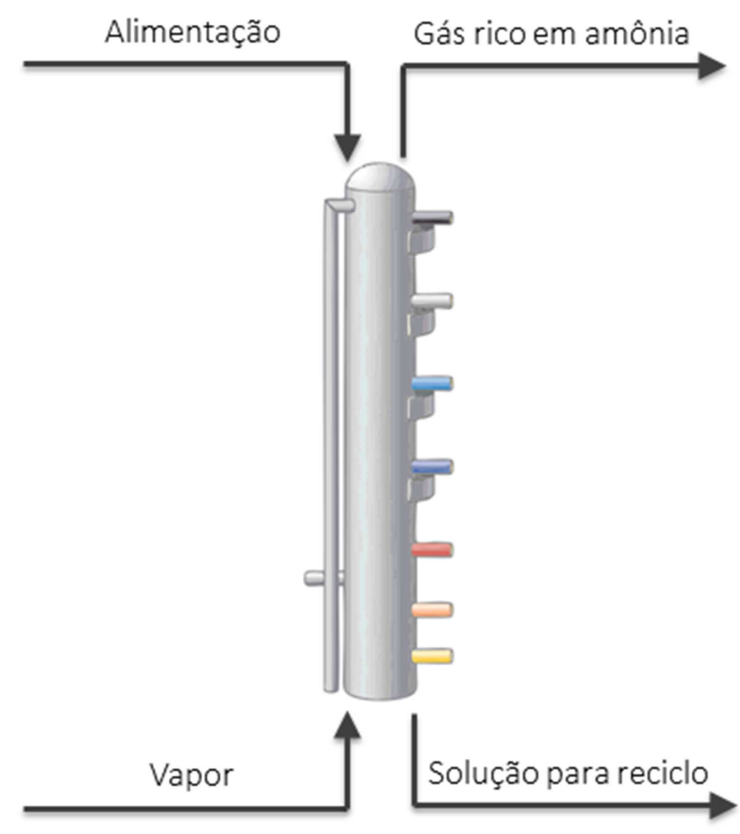

Figura 1. Esquemático de funcionamento de uma torre de recuperação de amônia.

Para controlar a alimentação de solução e de vapor da torre, é necessário manter as temperaturas de topo e de fundo em faixas pré-determinadas de operação. $O$ sistema de controle não pode permitir uma operação com: (i) baixas temperaturas, pois gera contaminação de amônia na base do equipamento, isto é, perda de amônia ou (ii) altas temperaturas, pois gera acúmulo de resíduos no equipamento, aumentando a pressão interna das torres e o número de paradas para limpeza.

A pressão e vazão do vapor vivo possuem variações em seu fornecimento devido às características de operação da planta, gerando distúrbios na temperatura do equipamento. Todas estas particularidades apresentadas acima tornam a estratégia de controle automático tradicional (PID) não muito eficiente $\mathrm{e}$, por isso, os operadores realizavam intervenções no controle constantemente. Dessa forma, era consumido mais vapor do que o necessário. 


\section{MATERIAIS E MÉTODOS}

\subsection{Descrição da Unidade T-31 de Recuperação de Amônia}

Na unidade T-31 de Niquelândia, a recuperação de amônia é realizada em seis torres em paralelo, seguindo um rodízio de lavagem e operação: das seis torres, duas ou mais estarão sempre em procedimento de lavagem, enquanto as outras continuam operando. Este procedimento ocorre frequentemente, na medida em que as incrustações provenientes dos compostos presentes na solução amoniacal vão se acumulando ao longo do tempo, gerando pressurização na torre.

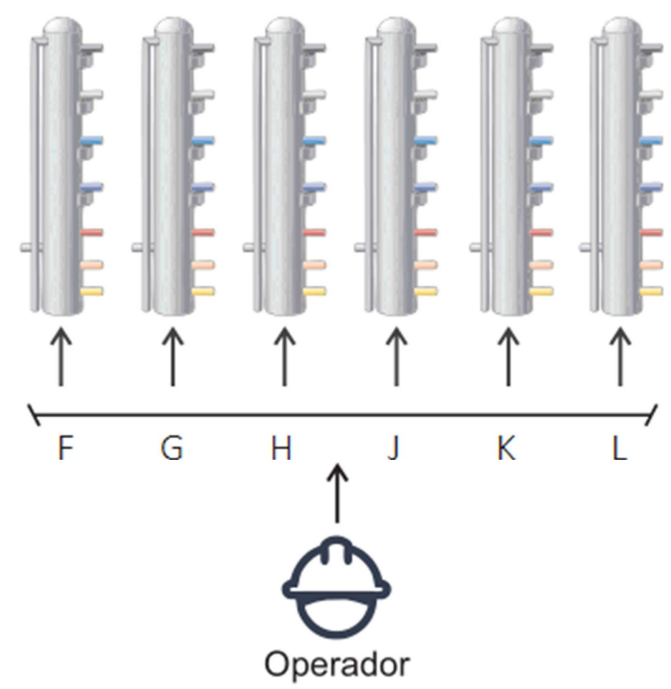

Figura 2. Representação da operação manual das torres de amônia.

Para cada torre, existem dois controladores: um para a alimentação e outro para o vapor. As exceções são as torres $\mathrm{G} \mathrm{e} \mathrm{K}$, pois cada uma possui 3 controladores: um para a alimentação e os outros dois permitem o chaveamento entre as duas linhas de vapor (torre $\mathrm{G}$ pode receber vapor tanto da linha $\mathrm{F}$ quanto da linha $\mathrm{H}$; torre $\mathrm{K}$ pode receber vapor tanto da linha $\mathrm{J}$ quanto da linha $\mathrm{L}$ ).

\subsection{Conceitos Sobre Lógica Fuzzy}

A lógica Fuzzy permite que estados indeterminados, como "muito baixo", "frio", "morno", etc., possam ser quantificados e processados por um computador. Quando se lida com problemas de categorização de conceitos, surge a dificuldade em ver distinções claras entre os estados ou as qualificações, gerando os problemas de classificação.

Por exemplo, tem-se um tanque de água e a informação humana sobre o nível de água neste reservatório. Dentro dos estados possíveis (baixo, alto, médio, etc.), existe uma transição que pode ser apresentada como um gradiente, no qual não é possível afirmar com certeza onde um estado termina e onde outro começa. Entretanto, é possível categorizar um valor numérico de nível como algo que pertence a mais de um estado ao mesmo tempo. Supondo que o tanque tenha $4 \mathrm{~m}$ de altura e o nível de água esteja em $1 \mathrm{~m}$ de altura a partir do chão, a variável "nível" pode ser classificada como $75 \%$ baixa e $25 \%$ alta, por exemplo. Estas porcentagens são chamadas de funções de pertinência e a partir delas é possível construir lógicas 
computacionais que sejam capazes de mapear funções que descrevem processos reais. Para que o mapeamento seja razoável, é necessário utilizar um número considerável de funções de pertinência.

Em controle de processos químicos, uma malha de controle fechada monitora as variáveis de processo e calcula o erro destas variáveis a partir dos setpoints informados ao controlador. Essa diferença entre o valor real e o valor desejado gera um sinal de resposta que é informado ao atuador, para que ele gere uma mudança no processo de forma que a variabilidade seja reduzida ao longo do tempo.

É possível aplicar a lógica Fuzzy para avaliar com maior precisão a resposta que deve ser dada ao atuador, ao incorporar a lógica humana de como tratar estados indeterminados. Neste cenário, a estratégia a ser adotada é incorporar as regras de controle do operador responsável pelo processo, visto que este acompanha a operação em diversas situações, e a do engenheiro de processos, que possui informações relacionadas ao projeto da operação unitária. Além disso, o controlador não possui limitações com relação ao número de variáveis e consegue incorporar não linearidades características de processos químicos.

Para desenvolver um controlador utilizando lógica Fuzzy, é necessário classificar as variáveis de processo (PV), manipuláveis (MV) e de distúrbio (DV) em rótulos que indiquem intensidade e direção com relação ao setpoint de processo. Estes rótulos irão definir intervalos de valores de resposta para atuadores e são representadas por triângulos em um plano cartesiano, como ilustrado na Figura 3.

Seguindo o exemplo do nível de água no tanque, o controlador irá rotular a variável de entrada como $40 \%$ baixo e $60 \%$ muito baixo. Considerando que existe uma válvula que regula a entrada de água no tanque, é possível aplicar o mesmo raciocínio de classificação para a variável "abertura da válvula".

O controlador Fuzzy irá acionar rótulos de saída a partir das variações de nível em relação ao setpoint, em um processo chamado de fuzzyficação. Cada associação entre o rótulo de entrada e de saída é chamado de regra Fuzzy e mais de uma regra pode ser acionada ao mesmo tempo. Dessa forma, a saída para o atuador tem diferentes rótulos com diferentes intensidades e passa por um processo chamado de defuzzyficação, no qual o centro de massa da união da área dos triângulos acionados resulta em uma saída de controle de valor único. Com relação ao exemplo do tanque, o sinal de entrada lido aciona o rótulo "grande", com intensidade de $35 \%$, e "muito grande", com valor de $75 \%$, gerando um valor final de abertura de $58 \%$. 


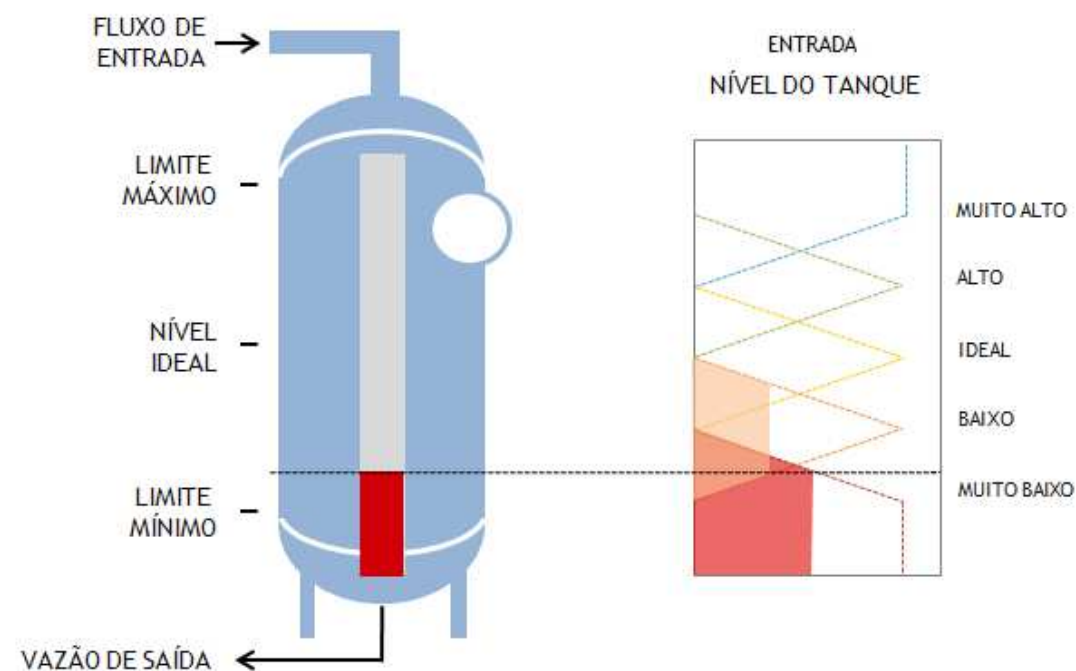

SAIDA

ABERTURA DA VÁLVULA

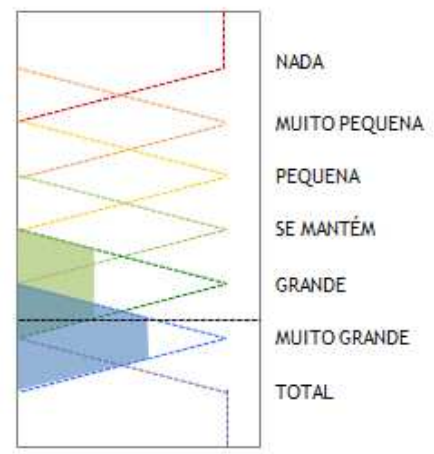

Figura 3. Exemplo de aplicação de rótulos em variáveis de processo.

O controlador se torna mais preciso na medida em que mais regras Fuzzy são utilizadas, permitindo a descrição de comportamentos de processos mais complexos.

\subsection{Metodologia de controle}

A plataforma de controle avançado Leaf ("Learning Fuzzy"), baseada em lógica Fuzzy, foi desenvolvida pela I.Systems e aplicada nas torres de recuperação de amônia como ferramenta na criação de controladores de vapor e de solução. A etapa de implantação, que seguiu desde a análise de infraestrutura e automação, até o momento em que o Leaf foi habilitado, é descrita abaixo:

1) Avaliação e adaptação da estrutura de automação e do SDCD (2 dias): criação de dois pares independentes de botões habilita/desabilita Leaf em cada torre - um aciona o controle de alimentação de solução e o outro aciona o controle de vapor na base da torre - e inserção de lógica de segurança para situações de falhas de comunicação, monitorada por um sinal do tipo watchdog que emite pulsos enquanto houver comunicação (2 dias);

2) Criação de estratégia de controle para os controladores de vapor e de alimentação de solução da torre $\mathrm{H}$, além da sintonia dos mesmos e realização de testes de robustez ( 3 dias);

3) Adaptação das estratégias de controle para as outras torres (5 dias); e

4) Ativação do Leaf.

Após o período de comissionamento, o controle da unidade foi alternado a cada 3 dias entre: operação com Leaf ligado e operação manual, pelo período de 1 mês. Este período foi denominado como período de avaliação e teve como objetivo coletar dados históricos para comparação do benefício gerado, de forma que os mesmos distúrbios diários fossem aplicados aos dois cenários.

A equipe de automação realizou a criação de dois pares independentes de botões habilita/desabilita Leaf em cada torre, sendo um botão para acionar o controle da alimentação de solução e outro botão para o acionamento do controle de vapor na base da torre. A Figura 4 apresenta a tela de operação para uma das torres. As telas das outras torres são similares e possuem os mesmos botões. 


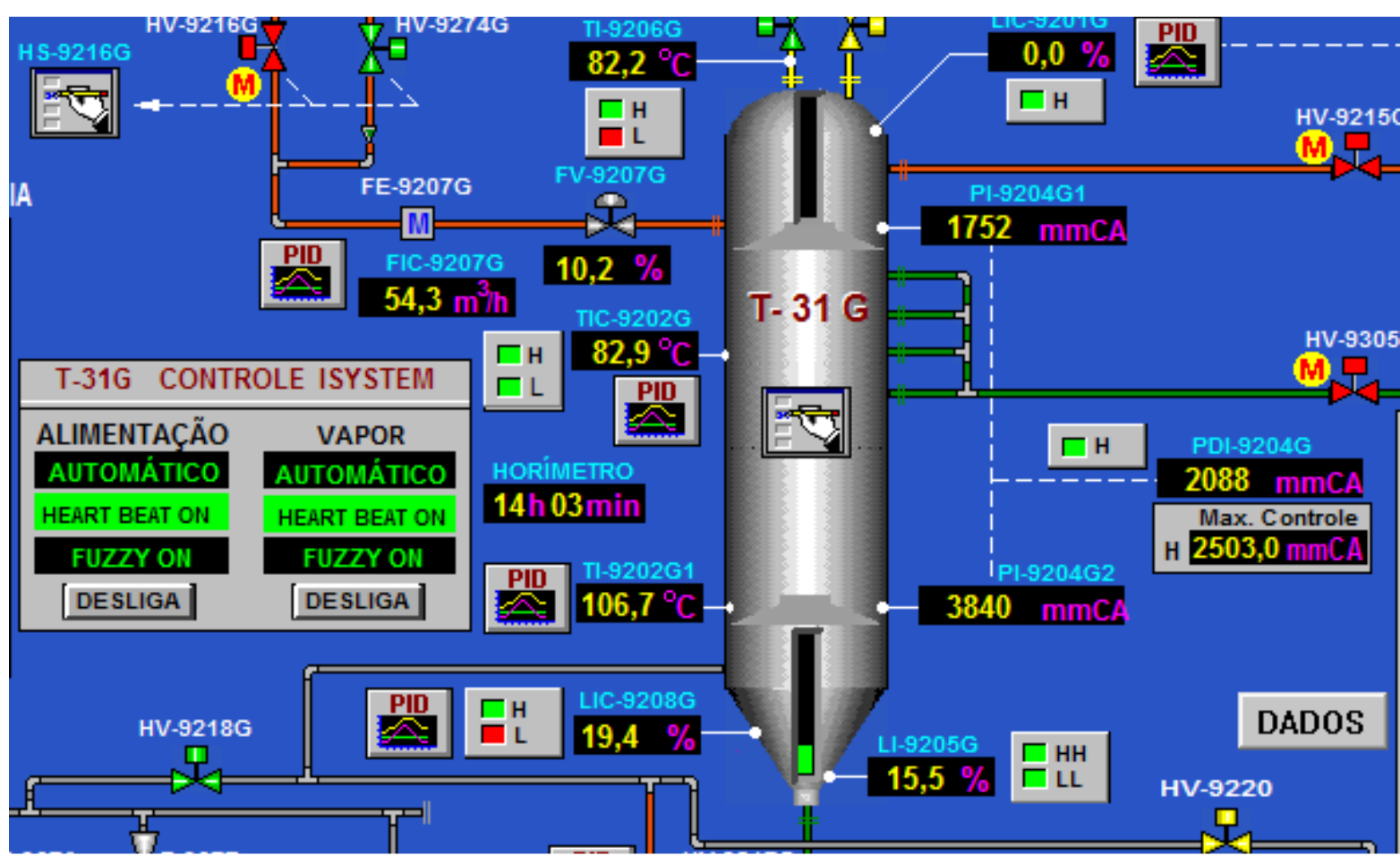

Figura 4. Interface da operação com o acionamento do Leaf para controle das torres.

Ainda na mesma tela, é possível ver os avisos relacionados ao status da comunicação OPC. Em caso de falha de comunicação, o aviso HEART BEAT OFF substituirá o aviso HEART BEAT ON, conforme Figura 5 abaixo:

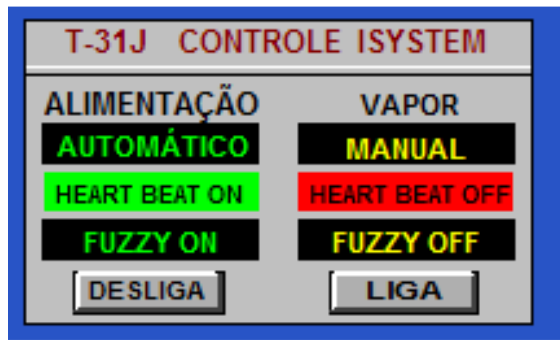

Figura 5. Aviso de falha de comunicação na tela de operação de uma das torres.

Em caso de falha de comunicação, o Leaf desabilitará automaticamente e só retornará após o operador habilitá-lo novamente.

É possível que o operador altere os parâmetros utilizados no controle, ao clicar no botão do PID que controla a válvula de vapor e digitar o mínimo e máximo para a abertura da mesma, de acordo com a Figura 6: 


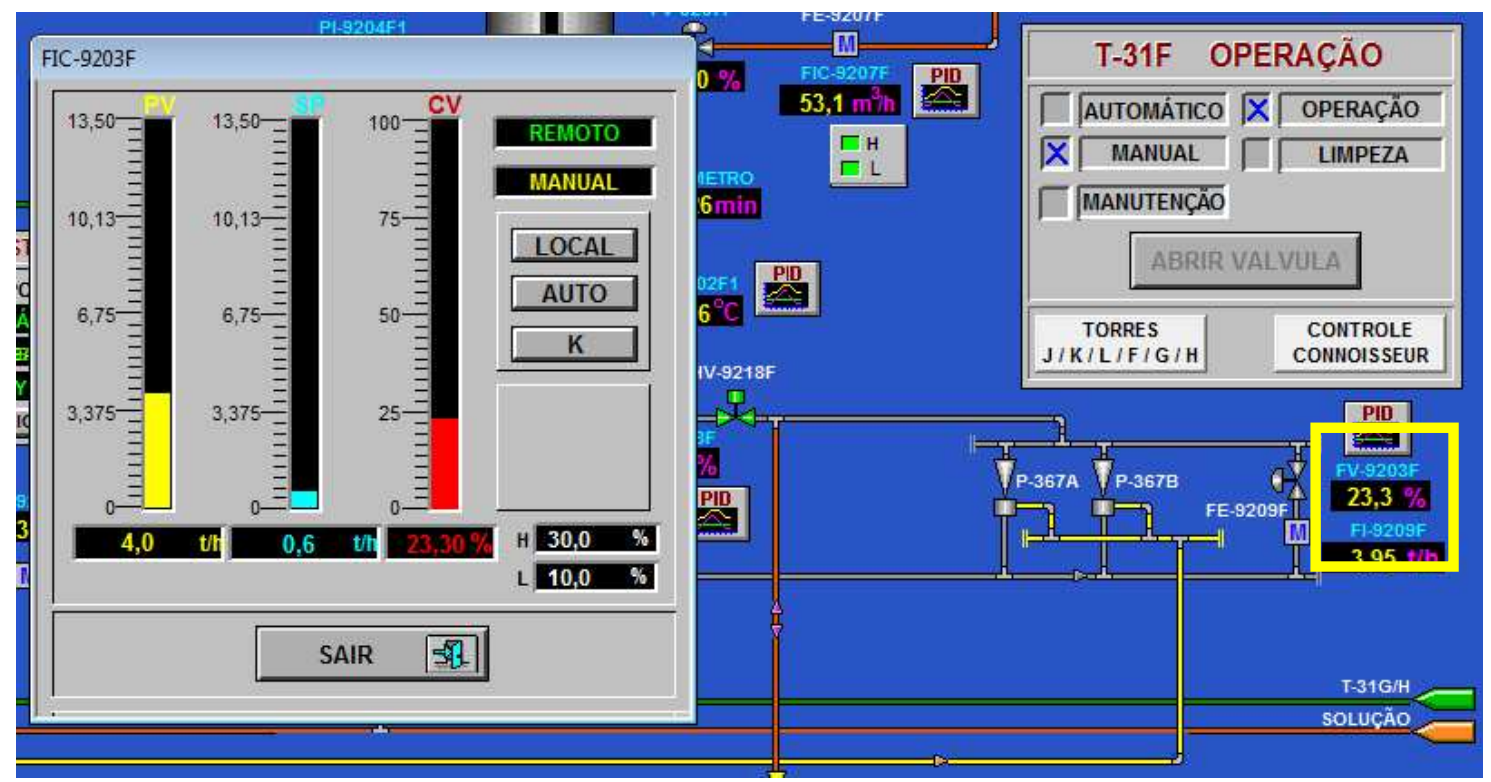

Figura 6. Configuração dos parâmetros para o controle da válvula de vapor na tela de operação.

Para o controle de alimentação, é possível configurar o mínimo, máximo e o set point da temperatura de topo da coluna. Ao pressionar o botão do PID que controla a alimentação, a janela de configuração é aberta, como ilustra a Figura 7:

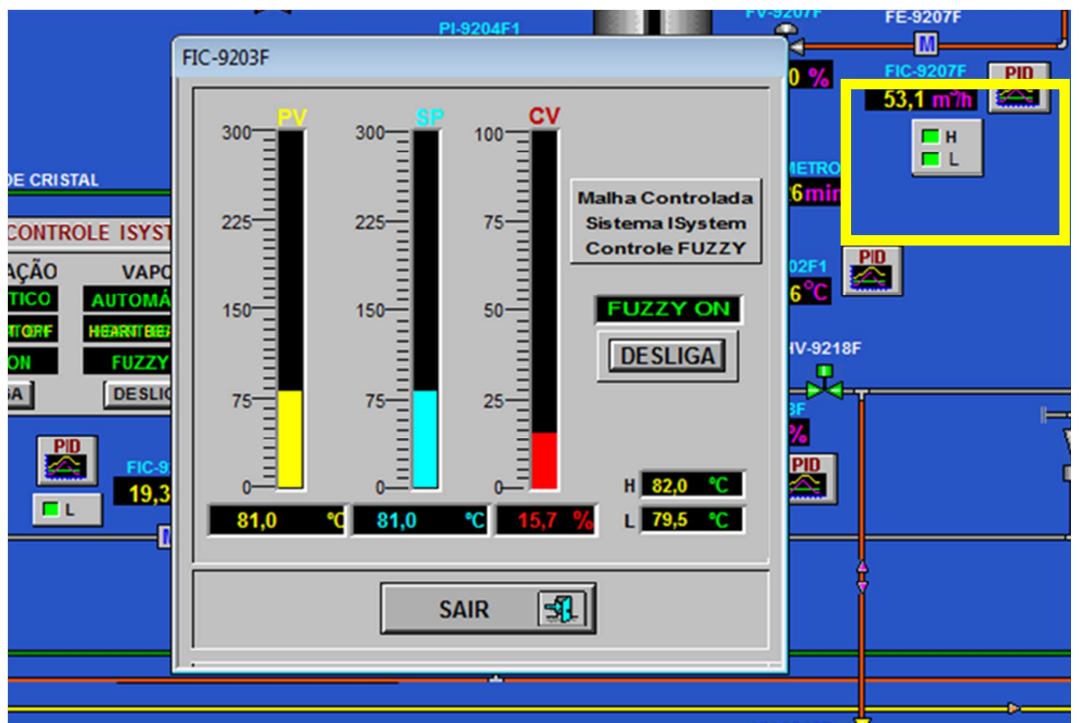

Figura 7. Configuração dos parâmetros para o controle da alimentação na tela de operação.

O chaveamento entre as linhas de vapor nas torres $G$ e $K$ é feito usando a informação do supervisório para acionar a linha de vapor utilizada, conforme Figura 8 abaixo: 


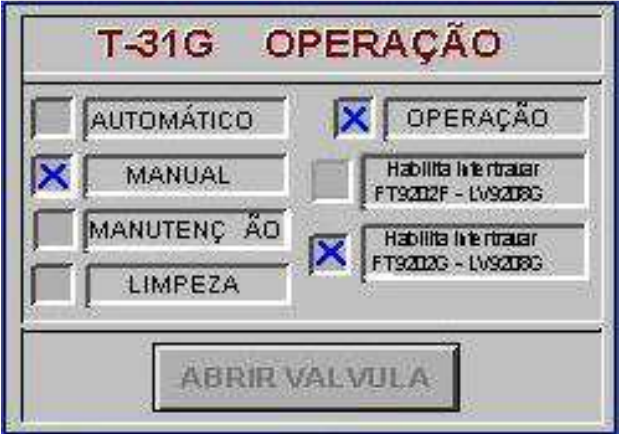

Figura 8. Controle de vapor entre as torres G e K.

As variáveis historiadas pelo Leaf estão presentes na Tabela 1:

Tabela 1. Variáveis historiadas pelo Leaf.

\begin{tabular}{|c|c|c|}
\hline Variável & Unidade & Captura \\
\hline Temperatura de topo & ${ }^{\circ} \mathrm{C}$ & Instrumento de campo \\
\hline Máximo da temperatura de topo & ${ }^{\circ} \mathrm{C}$ & Supervisório \\
\hline Mínimo da temperatura de topo & ${ }^{\circ} \mathrm{C}$ & Supervisório \\
\hline Set point da temperatura de topo & ${ }^{\circ} \mathrm{C}$ & Supervisório \\
\hline Diferencial de pressão da torre & $\mathrm{mmCA}$ & Instrumento de campo \\
\hline $\begin{array}{l}\text { Máximo do diferencial de pressão da } \\
\text { torre }\end{array}$ & $\mathrm{mmCA}$ & Supervisório \\
\hline Alimentação de solução & $\mathrm{m}^{3} / \mathrm{h}$ & Instrumento de campo \\
\hline Mínimo da alimentação da solução & $\mathrm{m}^{3} / \mathrm{h}$ & Supervisório \\
\hline Máximo da alimentação da solução & $\mathrm{m}^{3} / \mathrm{h}$ & Supervisório \\
\hline Abertura da válvula de vapor & $\%$ & Instrumento de campo \\
\hline Mínimo da abertura da válvula de vapor & $\%$ & Supervisório \\
\hline $\begin{array}{l}\text { Miaxımo da abertura da valvula de } \\
\text { vapor }\end{array}$ & $\%$ & ervisório \\
\hline Temperatura de base & ${ }^{\circ} \mathrm{C}$ & Instrumento de campo \\
\hline Mínimo da temperatura de base & ${ }^{\circ} \mathrm{C}$ & Supervisório \\
\hline Máximo da temperatura de base & ${ }^{\circ} \mathrm{C}$ & Supervisório \\
\hline Set point da temperatura de base & ${ }^{\circ} \mathrm{C}$ & Supervisório \\
\hline Pressão do vapor (utilidades) & $\mathrm{kgf} / \mathrm{cm}^{2}$ & Instrumento de campo \\
\hline Vazão de vapor & $t / h$ & Instrumento de campo \\
\hline Vazão de alimentação & $\mathrm{m}^{3} / \mathrm{h}$ & Instrumento de campo \\
\hline
\end{tabular}

\subsubsection{Controle de alimentação}

A malha de alimentação de solução é ilustrada pela Figura 9 abaixo. A PV do controle é a temperatura de topo e os valores de mínimo, máximo e setpoint são controlados pela operação. Ela deve estar sempre dentro dos limites estabelecidos por questões de segurança da fábrica. A relação entre a temperatura de topo e a vazão de alimentação é direta: quando a temperatura começa a subir, adiciona-se mais solução para que o perfil de temperatura da torre esfrie alguns graus.

O diferencial de pressão da torre foi adicionado como DV e possui relação inversa com a vazão de alimentação. É necessário também manter a pressão de operação dentro dos limites especificados. 


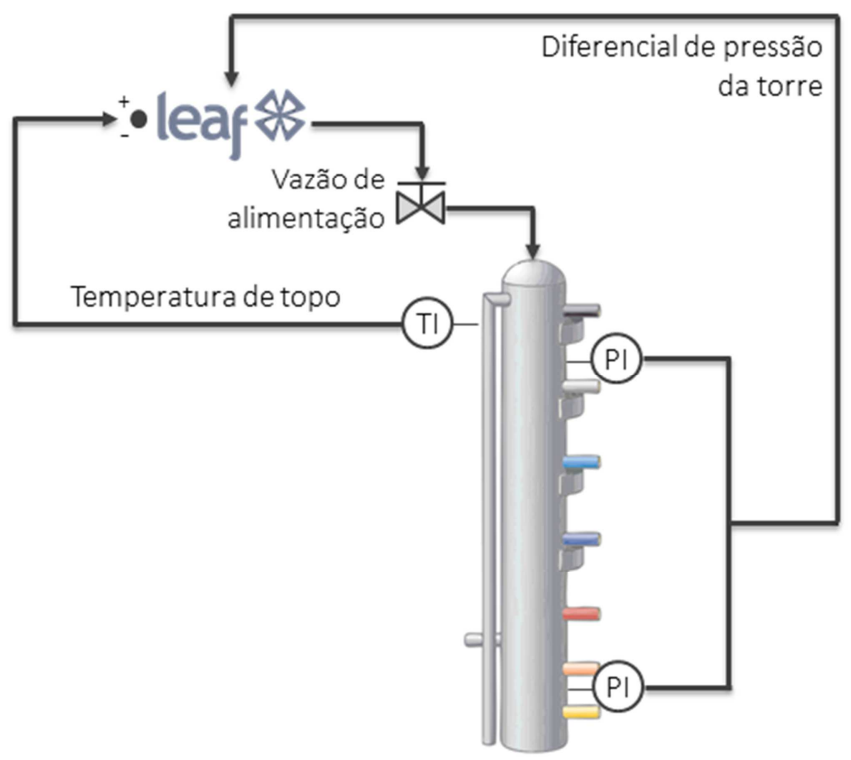

Figura 9. Fluxograma da malha de controle de solução.

A referência da MV (abertura da válvula de alimentação) é a média entre os limites de máximo e mínimo. O Leaf traz um módulo de controle integrativo característico de controles do tipo feedback ${ }^{1}$, acoplado com tecnologia de anti-windup ${ }^{2}$ para evitar saturação durante o cálculo do acúmulo de erro, com o objetivo final de eliminar o offset $^{3^{3}}$ do sistema. $O$ integrativo foi ajustado de forma que ele possa agir rapidamente, pois este controlador requer um ajuste fino e preciso da temperatura de topo.

Por fim, para eliminar ruídos característicos de sinais de válvula, adicionou-se um filtro exponencial na saída do controlador.

\subsubsection{Controle de vapor}

A malha de injeção de vapor é ilustrada pela Figura 10 abaixo. Similar ao controle de alimentação, o controle de vapor também utiliza a temperatura de topo como PV. Durante o comissionamento, a equipe de operação solicitou que o controle de vapor fosse mais lento que o de alimentação, para que ele seja acionado somente quando a vazão de alimentação não fosse mais suficiente para ajustar a temperatura do topo.

\footnotetext{
${ }^{1}$ Controle feedback, ou por realimentação: é o procedimento no qual parte do sinal de saída retorna para a entrada do sistema. Em controle de processos, o erro é calculado como a diferença entre o sinal de saída atual e o setpoint desejado.

${ }^{2} \mathrm{O}$ fenômeno de windup ocorre quando o controlador apresenta saturação do sinal de controle acelerado pela ação integral do mesmo. Uma ferramenta com tecnologia de anti-windup previne a saturação do sinal de saída.

${ }^{3}$ Offset é o erro permanente entre a PV e o SP, após estabilização do processo. Em geral, o controlador com ação integrativa elimina esse desvio.
} 


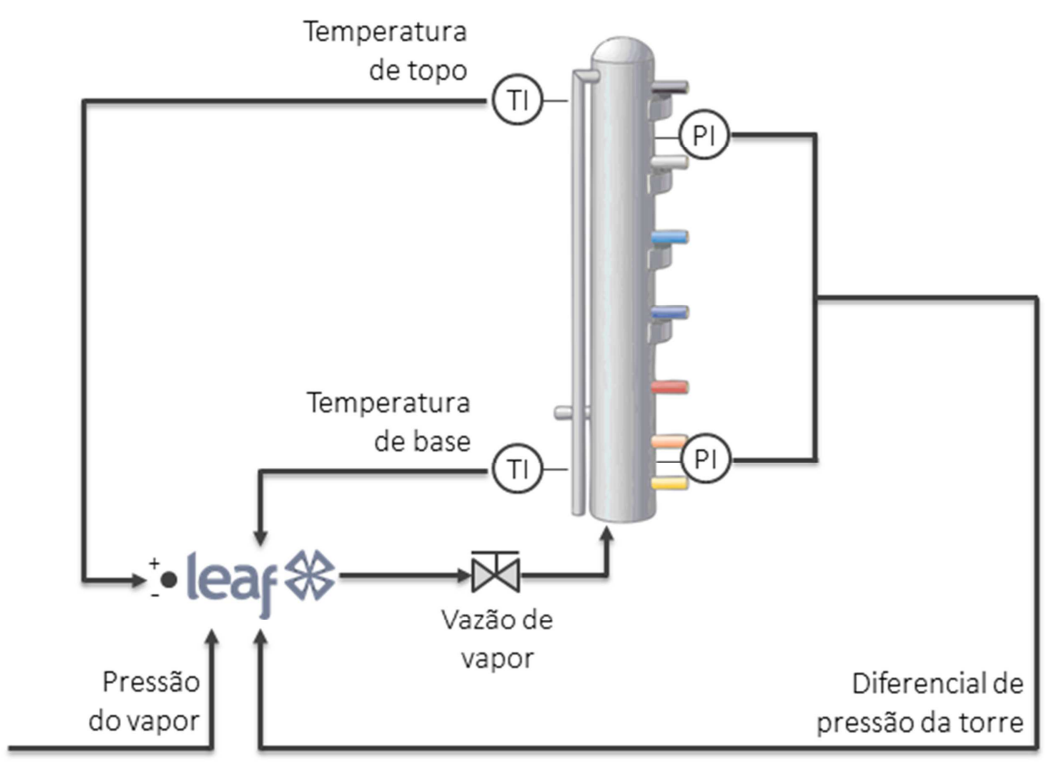

Figura 10. Fluxograma da malha de controle de vapor.

Isso justificou a escolha das influências para a PV e para as DVs da malha. Estas DVs (diferencial de pressão da torre, pressão do vapor e temperatura da base) possuem relação inversa com a vazão de vapor. Os limites e o setpoint da temperatura de base da torre podem ser ajustados pela operação.

A MV (abertura da válvula de vapor) possui os limites estabelecidos pela operação e o set point é calculado como a média destes limites. A não-linearidade da malha foi configurada de maneira que o comportamento da malha seja menos reativo que a de alimentação. O integrativo está menos intenso, para que a maior parte da correção seja feita no ajuste da vazão de alimentação.

Ao final, para eliminar ruídos característicos de sinais de válvula, adicionou-se um filtro exponencial na saída do controlador.

\section{RESULTADOS E DISCUSSÃO}

O sistema de controle avançado Leaf controlou a temperatura de topo, reduzindo o vapor quando possível e mantendo a temperatura controlada no setpoint desejado, com erro de $\pm 0.5^{\circ} \mathrm{C}$. É possível observar que o sistema manteve a estabilidade da torre, mesmo em estado pressurizado, conforme Figuras 11 e 12 abaixo.

Ainda a partir da Figura 12, nota-se que mesmo com a mudança de setpoint de processo, o Leaf respondeu rapidamente e manteve a variável próxima do valor desejado. Durante estes testes de robustez, o Leaf encontrou um balanço mantendo o delta de pressão da torre em torno de $2.400 \mathrm{mmCA}$ e uma temperatura em torno de $80^{\circ} \mathrm{C}$. Ao tornar o processo mais estável, o Leaf reduziu a temperatura média das torres de 1 a $2^{\circ} \mathrm{C}$, sem gerar contaminação de amônia na base. O controlador realizou o balanço de vazões de alimentação e de vapor, de forma a priorizar a eficiência no consumo específico, para obter uma correção eficaz nos distúrbios que afetam a temperatura. 
Temperatura ${ }^{\circ} \mathrm{C}$

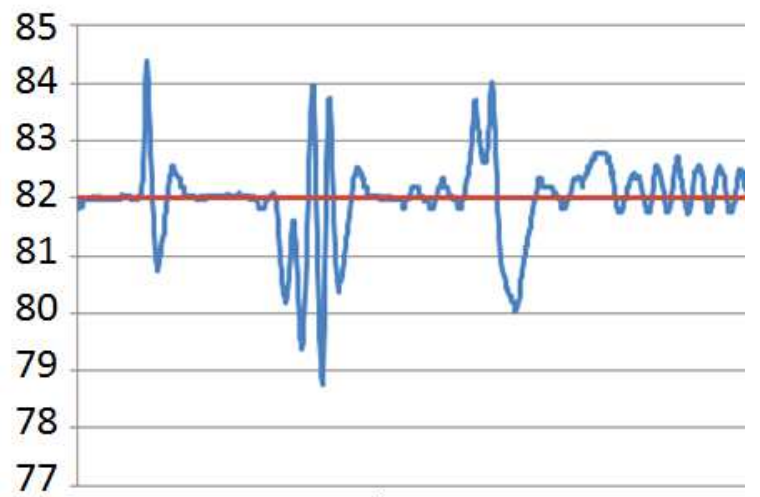

14 horas

Figura 11. Temperatura de topo da torre $\mathrm{F}$ operando por $14 \mathrm{~h}$ sem o Leaf.
Temperatura ${ }^{\circ} \mathrm{C}$

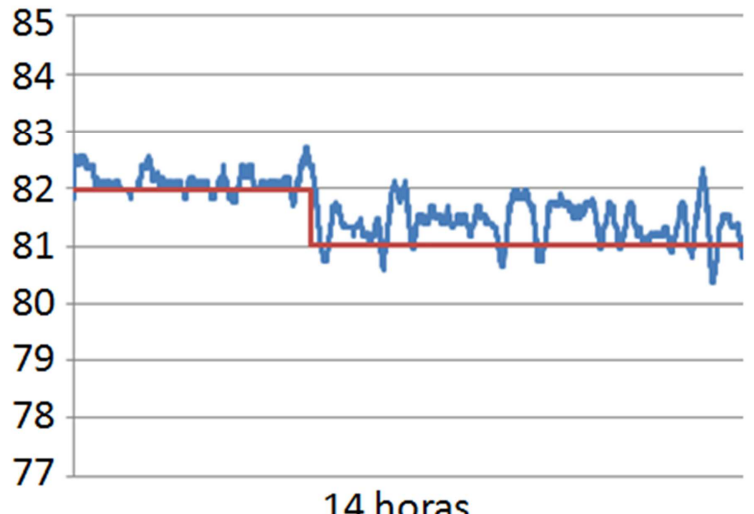

14 horas

Figura 12. Temperatura de topo da torre $\mathrm{F}$ operando por $14 \mathrm{~h}$ com o Leaf.

Com a redução do perfil de temperatura da torre, uma economia de $3,5 \%$ no consumo específico de vapor foi gerada, sem afetar a produtividade da unidade de recuperação de amônia. Além disso, como a operação dos fluxos de vapor e de alimentação foi automatizada, reduziu-se a intervenção manual em consequência. Este é um benefício importante, visto que a ferramenta de controle avançado monitora as variáveis a cada segundo, portanto as correções são feitas em tempo real.

\section{CONCLUSÃo}

O sistema de controle avançado foi capaz de gerar benefício ao economizar vapor, mantendo o perfil de temperatura das torres dentro dos valores desejados com menor variabilidade, se comparado ao controle realizado com intervenção manual constante. O Leaf é capaz de tratar a não linearidade do processo de destilação por arraste de forma adequada, além de levar em consideração várias informações ao cálculo simultaneamente (controle multivariável).

\section{Agradecimentos}

Agradecemos a equipe Votorantim e I.Systems contribuiu com este trabalho dedicando tempo, dedicação e conhecimento.

\section{BIBLIOGRAFIA}

1 Rodrigo Alberto M. Gomes. Aumento da recuperação de Níquel, Cobalto e Cobre nas etapas de pirometalurgia e hidrometalurgia da Votorantim Metais Níquel - Niquelândia. Dissertação de Mestrado. Universidade Federal de Minas Gerais - Belo Horizonte/MG.

2 Isenmann F. de Armin. Destilação, retificação e arraste por vapor. CEFET-MG, Timóteo/MG

3 Fernando Gomide e Ricardo Gudwin. Modelagem, Controle, Sistemas e Lógica Fuzzy. Revista SBA: Controle \& Automação, vol 4, n 3, 1994. 